\title{
Critical Exponents for Metal-Insulator Transition in Two-Dimensional Systems
}

\author{
J. WOJTKIEWICZ* \\ Department for Mathematical Methods in Physics \\ Faculty of Physics, University of Warsaw \\ Hoża 74, 00-682 Warszawa, Poland \\ (Received November 13, 2008; in final form December 30, 2008)
}

\begin{abstract}
Three years ago, a new universality class, associated with the metal-insulator transition in quasi-two-dimensional compounds, was discovered. Imada has given explanation of the observed critical behaviour. Considerations in this work are based on the assumption that the one-particle dispersion is quartic one instead of the standard quadratic behaviour. In this paper, it is shown that other possible natural non-standard dispersions lead to other possible critical behaviour and critical exponents.
\end{abstract}

PACS numbers: 71.30.+h, 71.10.Fd, 02.40.Xx

\section{Introduction}

The metal-insulator transition (MIT) takes place, when a given system undergoes a transition from the conductor state (characterised by conductivity $\sigma>0$ ) to the insulator state $(\sigma=0)$ under change of certain external parameter, for instance the pressure. Rigorous definition of the MIT can be given within the linear response theory $[1,2]$. The difference between conductor and metal can be precisely defined only at zero temperature.

The MIT phenomenon is known for quite a long time. There are known various kinds of this phenomenon, differing by their properties and underlying mechanisms $[1,2]$.

As a canonical example of the MIT one can mention this phenomenon in (doped) vanadium oxide $\mathrm{V}_{2} \mathrm{O}_{3}[3,4]$. Under growing pressure, the compound undergoes transition from the insulator state to the conductor. At present time, this phenomenon is well understood: There is general consensus that this transition belongs to universality class of the 3-dimensional Ising model, characterised by critical exponents $\delta \approx 4.9, \beta \approx 0.33, \gamma \approx 1.25$ [4]. The critical temperature is high (about $500 \mathrm{~K}$ ), and satisfactory description of the transition within renormalisation group based on the Landau-Ginzburg-Wilson (LGW) functional has been proposed. It has been conjectured that "this is potentially a generic description of the Mott critical endpoint in correlated electron materials" [5].

In such a situation, the discovery of different critical behaviour in MIT was quite surprising. In 2005 , the MIT in organic salt $(\mathrm{BEDT}-\mathrm{TTF})_{2} \mathrm{X}$ (bis-

\footnotetext{
* e-mail: wjacek@fuw.edu.pl
}

-etylenoditio-tetratiofulvalene; $\mathrm{X}$ is an anion, for instance $\mathrm{Cu}\left[\mathrm{N}(\mathrm{CN})_{2}\right] \mathrm{Cl}$ ) was discovered under change of the ambient pressure [6]. (BEDT-TTF $)_{2} \mathrm{X}$ is a compound possessing a layered, quasi-two-dimensional structure. The MIT takes place at low temperatures (the critical temperature $T_{\mathrm{c}} \approx 40 \mathrm{~K}$ ) and quantum effects play an important role in this transition - it can serve as an example of the quantum critical point [6-9].

In the neighbourhood of the critical pressure $p_{\mathrm{cr}}$, the conductivity $\sigma$ has the following form:

$$
\sigma \sim\left(p-p_{\text {cr }}\right)^{1 / \delta} .
$$

In the physics of magnetism, we have analogous formula

$$
M \sim H^{1 / \delta}
$$

( $M$ - magnetisation, $H$ - magnetic field).

Critical exponents, associated with MIT in (BEDT$-\mathrm{TTF})_{2} \mathrm{X}$ salt are [6]:

$$
\delta=2, \quad \beta=1, \quad \gamma=1 .
$$

Such values have not been observed in other phase transitions! In the other words, new universality class have been discovered. (What is also surprising, this is the anomally low value of the $\delta$ exponent. In magnetic physics, the lowest value of $\delta$ is 3 in the case of mean field theory; in other situations $\delta>3$.)

The convincing explanation has been proposed soon by Imada [10]. It is based on the Landau-Ginzburg-Wilson approach modified in such a way that the description of the neighbourhood of the quantum critical point is possible.

The Imada explanation is based on one-particle description of the system; so, it can be viewed as a meanfield treatment. However, Imada reasoning goes behind such an approach: he asks why such a mean-field treat- 
ment gives so good description of the critical behaviour of the system. He gives a lot of arguments supporting correctness of the MFT-like description in the critical region, based mainly on certain results concerning the MIT in the extended two-dimensional Hubbard model, scaling theory for Mott transitions, and the analysis of one-particle quantum dynamics. Moreover, Imada examines validity of such a mean-field picture in description of the MIT of BEDT-TTF salts.

The one-particle model of the MIT, used by Imada, can be considered as a variant of the Bloch-Wilson model $[1,2]$. Its main assumptions are as follows:

1. The one-particle model is used (i.e. the HartreeFock approximation gives good description).

2. Two energy bands are present. The lower one is filled and the upper one is empty, and

3. under change of external parameter (such as pressure $p$ ) the upper band is lowered down and beginning from some critical value of parameter $p_{\mathrm{cr}}$, its filling becomes non-zero. It is illustrated in Fig. 1.

4. The upper band possess the "degenerate" dispersion, i.e. in the Taylor expansion of the $E(\boldsymbol{k})$, quadratic terms are absent. Imada assumed the quartic dispersion: $E(\boldsymbol{k}) \sim\left(|\boldsymbol{k}|^{2}\right)^{2}$ instead of standard quadratic one: $E(\boldsymbol{k}) \sim|\boldsymbol{k}|^{2}[10]$.

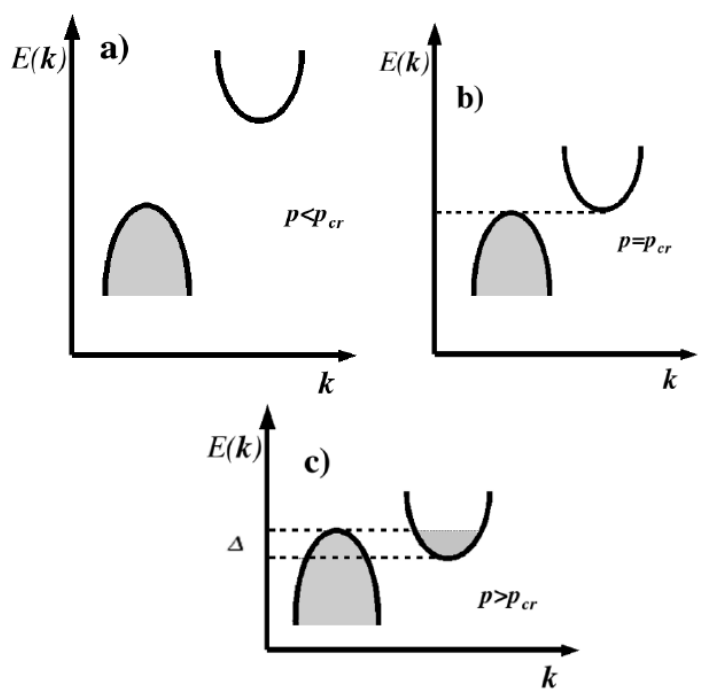

Fig. 1. Schematic picture of the Bloch-Wilson transition: (a) the system in insulating phase; (b) critical point; (c) the system in conductor phase.

The "degenerate" dispersion (corresponding to the degenerate minimum of $E(\boldsymbol{k})$, exemplified by 4$)$ above, is characteristic of frustrated systems. However, the form $E(\boldsymbol{k}) \sim\left(|\boldsymbol{k}|^{2}\right)^{2}$ considered by Imada, is not unique one: there are possible another asymptotic forms of degenerate $E(\boldsymbol{k})$ for $\boldsymbol{k} \approx \mathbf{0}$. For smooth $E(\boldsymbol{k})$, degenerate minima and their standard ("normal") forms have been classified by Arnol'd and Vassilyev [11].

In this paper, some simplest possible cases of $E(\boldsymbol{k})$ with degenerate minimum have been examined. The first group of them are degenerate anisotropic minima. For instance, in two-dimensional case, in one direction $E$ possess ordinary quadratic asymptotic, whereas in the second one, the asymptotic is quartic: $E(\boldsymbol{k}) \sim k_{1}^{2}+k_{2}^{4}$. Another examples considered possess asymptotic of $E(\boldsymbol{k})$ characteristic of one-particle spectrum in the Néel potential. At the energy band edge, the dispersion relation has the asymptotic form $E(\boldsymbol{k}) \sim K_{1}^{2} K_{2}^{2}$, where $K_{1}=k_{1}+k_{2} \pm \pi, K_{1}=k_{1}-k_{2} \pm \pi$. In both cases, the critical exponents $\delta, \beta, \gamma$ have been calculated.

The outline of the paper is as follows. In Sect. 2 , the $\delta$ exponent is calculated. In this case, the whole Imada reasoning can be simplified, and this simplified form is presented.

Other critical exponents $(\beta$ and $\gamma)$ are calculated in more complicated manner. Description of the neighbourhood of quantum critical point is needed, and the form of the free energy functional allowing such a description must be derived. Such a form has been proposed in [10] and is briefly summarised in Sect. 3. The $\beta$ and $\gamma$ are also calculated there.

Section 4 summarises the results obtained. Some open problems are also mentioned here as well as directions of some possible investigations.

\section{The $\delta$ exponent}

The $\delta$ exponent can be calculated by the following recipe:

1. The conductivity is proportional to the number of electrons $n_{\mathrm{e}}$ in the upper (conductivity) band.

2. This in turn can be obtained by integration of the density of states $D(E)$ :

$$
n_{\mathrm{e}}(\Delta) \sim \int_{0}^{\Delta} D(E) \mathrm{d} E,
$$

where $\Delta$ is the difference between the maximum of the lower band and the minimum of the upper band (see Fig. 1).

3. The asymptotics of density of states $D(E)$ can be calculated from dispersion relation $E(\boldsymbol{k})=$ $E\left(k_{1}, k_{2}\right)$ for the upper band by a straightforward calculation.

Remark. The asymptotic properties of $E(\boldsymbol{k})$ for $\boldsymbol{k} \approx \mathbf{0}$ (i.e. first terms of its expansion in a Taylor series) are crucial for the value of the $\delta$ exponent.

\section{The free energy functional and $\beta, \gamma$ exponents}

The standard LGW functional, used widely in (among other) magnetism physics and as a starting point in 
renormalisation-group calculations [12], has the following form:

$$
F=-M H+\frac{1}{2}\left(T-T_{\mathrm{c}}\right) M^{2}+\frac{b}{4} M^{4},
$$

where $F-$ free energy, $M-$ magnetisation, $H-$ magnetic field, $T$ - temperature. Let us notice that $F$ is analytic in $M, T, H$. Two parameters can be chosen as independent; perhaps the most often choice is $H$ and $T$.

After such a choice, the magnetisation $M(H, T)$ can be calculated from the condition

$$
\frac{\partial F}{\partial M}=0 \text {. }
$$

The function $M(H, T)$ gives critical exponents $\beta, \gamma, \delta$ by a standard calculation. Exponents obtained from the form (2) of the free energy take the classical (mean-field) values: $\delta=3, \beta=\frac{1}{2}, \gamma=1$.

The above form of the free energy functional is not adequate to description of the behaviour of the system in a neighbourhood of the quantum critical point [10]. Imada [10] has proposed the following form of the free energy functional.

We are considering the system of fermions in $d$ dimensions. Let $X$ be particle density, $\mu$ - the chemical potential (conjugated to $X$ ). The singular part of the free energy as a function of $\mu$ and $T$ is determined from equations

$$
\begin{aligned}
F & =-X \mu \\
& -\int \mathrm{d} E D(E) \ln (1+\exp (-(E-\mu) / T)), \\
X & =\int \mathrm{d} E f(E) D(E),
\end{aligned}
$$

where $D(E)$ - density of states, $f(E)$ - the FermiDirac distribution: $f(E)=[1+\exp ((E-\mu) / T)]$.

Remark. Expressions above are simple, but the justification, why they should be correct in the critical region, is quite intricate. For instance, in Imada arguments the quantum critical exponent appears, but it is not present in final formulas for other critical exponents.

The dispersion $E(\boldsymbol{k})$ is given by

$$
E(\boldsymbol{k})=a\left(g-g_{\mathrm{c}}\right)|\boldsymbol{k}|^{2}+\text { (h.o.t.), }
$$

where $a>0$, h.o.t. means "higher order terms", $g$ is a parameter driving the degeneracy of the minimum of $E$. For instance, as a parameter $g$ one can take the quotient $t^{\prime} / t$, where $t, t^{\prime}$ are amplitudes of nearest-neighbour and next-nearest-neighbour hoppings.

In the case $g \neq g_{\mathrm{c}}$, we have

$$
D(E) \sim E^{d / 2-1}
$$

and the case $g=g_{\mathrm{c}}$ corresponds to

$$
D(E) \sim E^{\theta},
$$

which gives the following form of the free energy functional:

$$
F=-X \mu-a\left(g-g_{\mathrm{c}}\right) X^{2 / d+1}+X^{\frac{\theta+2}{\theta+1}} .
$$

Let us observe that $F$ is non-analytic in $X$ !

An analogon of the magnetisation $M$ is the particle density $X$. Its form as a function of parameters
$X\left(\mu, g-g_{\mathrm{c}}\right)$ can be determined from an analogon of the condition (3):

$$
\frac{\partial F}{\partial X}=0 \text {. }
$$

The form (9) of the free energy functional gives the value of the critical exponents. The $\delta$ exponent is

$$
\delta=\frac{1}{\theta+1} \text {. }
$$

By a straightforward calculations, we obtain the following values of other critical exponents $\beta$ and $\gamma$ :

$$
\begin{aligned}
& \beta=\left(\frac{1}{\theta+1}-\frac{2}{d}\right)^{-1}, \\
& \gamma=1+\left(\frac{2}{d}-1\right) \beta .
\end{aligned}
$$

Values of these critical exponents are collected in Tables I and II for various asymptotics of $E(\boldsymbol{k})$.

It can be checked that the scaling relation

$$
\gamma=\beta(\delta-1)
$$

is always satisfied.

TABLE I

Some of simplest degenerate asymptotics of $E(\boldsymbol{k})$ and corresponding values of critical exponents: $d=2$. Values for standard (quadratic) asymptotics have been taken from [7] $(\delta=2 / d, \beta=d / 2, \gamma=1-d / 2)$. For remaining cases they have been calculated from formulae (10)-(12). ( $(\log )$ denotes "logarithmic correction".

\begin{tabular}{c|c|c|c|c|c}
\hline \hline$E\left(k_{1}, k_{2}\right)$ & $\rho(E) \sim E^{\theta}$ & $n_{E}(\Delta)$ & $\delta$ & $\beta$ & $\gamma$ \\
\hline$k_{1}^{2}+k_{2}^{2}[7]$ & const & $\Delta$ & 1 & 1 & 0 \\
$\left(k_{1}^{2}+k_{2}^{2}\right)^{2}[7]$ & $E^{-1 / 2}$ & $\Delta^{1 / 2}$ & 2 & 1 & 1 \\
$k_{1}^{4}+a k_{1}^{2} k_{2}^{2}+k_{2}^{4}$ & $E^{-1 / 2}$ & $\Delta^{1 / 2}$ & 2 & 1 & 1 \\
$k_{1}^{2}+k_{2}^{4}$ & $E^{-1 / 4}$ & $\Delta^{3 / 4}$ & $4 / 3$ & 3 & 1 \\
Néel, half-filling & $E^{-1 / 2} \log |E|$ & $\Delta^{1 / 2} \log |\Delta|$ & $2(\log )$ & $1(\log )$ & $1(\log )$
\end{tabular}

TABLE II

The same as in the previous table in $d=3$.

\begin{tabular}{c|c|c|c|c|c}
\hline \hline$E\left(k_{1}, k_{2}, k_{3}\right)$ & $\rho(E) \sim E^{\theta}$ & $n_{E}(\Delta)$ & $\delta$ & $\beta$ & $\gamma$ \\
\hline$k_{1}^{2}+k_{2}^{2}+k_{3}^{2}[7]$ & $E^{1 / 2}$ & $\Delta^{3 / 2}$ & $2 / 3$ & $3 / 2$ & $-\frac{1}{2}$ \\
$\left(k_{1}^{2}+k_{2}^{2}+k_{3}^{2}\right)^{2}[7]$ & $E^{-1 / 4}$ & $\Delta^{3 / 4}$ & $4 / 3$ & $3 / 2$ & $1 / 2$ \\
$k_{1}^{2}+k_{2}^{4}+k_{3}^{4}$ & const & $\Delta$ & 1 & 3 & 0 \\
$k_{1}^{2}+k_{2}^{2}+k_{3}^{4}$ & $E^{1 / 4}$ & $\Delta^{5 / 4}$ & $4 / 5$ & $\frac{15}{2}$ & $-\frac{3}{2}$
\end{tabular}

\section{Summary, open problems}

The critical exponents in the Bloch-Wilson kind of the MIT were considered. The critical behaviour is determined by the asymptotic form of the one-particle dispersion $E(\boldsymbol{k})$.

There are possible various forms of this asymptotics. This way, we obtain different forms of critical behaviour. 
Imada [10] examined the quartic asymptotics. But there are possible other natural asymptotics and corresponding sets of critical exponents. They have been calculated in the paper.

It would be very desirable to check experimentally if such possibilities are realised in nature.

The MIT in BEDT-TTF salts takes place in finite temperature $\left(T_{\mathrm{cr}} \approx 40 \mathrm{~K}\right)$. Imada considerations [10] as well as its extensions presented above concern the case $T=0$. It would be desirable to show that suitable modification of the Imada reasoning is applicable also to the finite temperature situation. An important step in this direction has been made in [13].

It is an intriguing question why an MFT-type approach, used by Imada (and in this paper), gives so good description of the MIT in the critical region. One should expect that the MFT picture will be strongly modified by correlation effects (we have to do with the correlationdriven phase transition!) Imada has given arguments why the MFT-type behaviour is observed even in the critical region. His arguments are convincing, but mainly qualitative. It would be extremely interesting to reproduce the observed MIT-type behaviour quantitatively within some concrete model, for instance, the Hubbard model with nn- and nnn hoppings (in such a way the frustration effects can be included).

Perhaps magnetic phase transitions can offer some hint. In the case of magnetic phase transition, the rule is that in realistic systems, the strong fluctuations in the critical region modify the mean-field behaviour, and observed critical exponents take values different from those predicted by MFT. It is a rule, but there are exceptions: There are known realistic systems, where critical behaviour is very well described by MFT [14]. The possible explanation of such a behaviour is that the system is close to the Kac limit (i.e. weak and long-range interactions). It would be again interesting to examine whether sources of observed MFT behaviour in two kinds of phenomena (MIT and magnetic transitions) have something in common or not.

The final remark is that the phase diagram of BEDT-TTF salts is quite rich $[6,10,13]$. Quite a lot of phases are present in various regions of phase diagram; there appear conductor, insulator, superconductor, ... It was possible to describe the neighbourhood of the critical point for MIT in a simple manner. But the situation complicates when one goes into the region where other phases are present. One can suspect connections and interplay of the MIT with other phase transitions in the system. Full description of the whole phase diagram will probably need much more complicated theory than presented in [10] and here.

\section{Acknowledgments}

I would like to thank the referee for useful remarks and comments.

\section{References}

[1] M. Imada, A. Fujimori, Y. Tokura, Rev. Mod. Phys. 70, 1039 (1998).

[2] F. Gebhard, The Mott Metal-Insulator Transition, Springer, Berlin 1997.

[3] D.B. McWhan, J.P. Remeika, Phys. Rev. B 2, 3734 (1970); D.B. McWhan, A. Menth, J.P. Remeika, W.F. Brinkman, T.R. Rice, Phys. Rev. B 7, 1920 (1973).

[4] H. Kuwamoto, J.M. Honig, J. Appel, Phys. Rev. B 22, 2626 (1980).

[5] P. Limelette, A. Georges, D. Jérome, P. Wzietek, P. Metcalf, J.M. Honig, Science 302, 89 (2003).

[6] F. Kagawa, K. Miyagawa, K. Kanoda, Nature (London) 436, 534 (2005).

[7] J. Spałek, A. Datta, J.M. Honig, Phys. Rev. Lett. 59, 728 (1987); J. Spałek, Postẹpy Fizyki (2006).

[8] J.A. Hertz, Phys. Rev. B 14, 1165 (1976).

[9] A.J. Millis, Phys. Rev. B 48, 7183 (1993).

[10] M. Imada, Phys. Rev. B 72, 075113 (2005).

[11] V.A. Vassilyev: Funkts. Anal. 11 (5), 3 (1977); V.I. Arnol'd, Varchenko, Gusein-Zade, Singularities of Differentiable Maps I, Birkhäuser, Boston 1985.

[12] J.J. Binney, N.J. Dowrick, A.J. Fisher, M.E.J. Newman, The Theory of Critical Phenomena. An Introduction to the Renormalization Group, Clarendon Press, Oxford 1992.

[13] T. Misawa, M. Imada, Phys. Rev. B 75, 115121 (2007).

[14] H. R. Ott, G. Keller, W. Odoni, Phys. Rev. B 25, $477(1982)$. 